\title{
Sensitive Potential Prognostic Markers for Colorectal Cancer Surgery Screened by UPLC-Q- TOF-MS Combined With Metabolomics Technology: UDP-D-Galactose
}

\author{
Zhao Zhang \\ Tianjin Union Medical Center \\ Lu Sun \\ Tianjin Medical University \\ Zhenying Zhao \\ Tianjin Union Medical Center \\ Weihua Zhang \\ Tianjin Union Medical Center \\ Lizhong Zhao \\ Tianjin Union Medical Center \\ Chen Xu \\ Tianjin Union Medical Center \\ Yuwei Li \\ Tianjin Union Medical Center \\ Yongjun Yu \\ Tianjin Union Medical Center \\ Chao Chen \\ Tianjin Union Medical Center \\ Mingsen Li \\ Tianjin Union Medical Center \\ Xipeng Zhang \\ Tianjin Union Medical Center \\ Linyi Dong ( $\nabla$ donglinyi@tmu.edu.cn ) \\ Tianjin Medical University
}

\section{Research Article}

Keywords: Colorectal cancer, UPLC-Q-TOF-MS, Biomarkers, UDP-D-galactose

Posted Date: April 15th, 2021 
DOl: https://doi.org/10.21203/rs.3.rs-396951/v1

License: (c) (1) This work is licensed under a Creative Commons Attribution 4.0 International License. Read Full License 


\section{Abstract}

Background: Colorectal cancer (CRC) is a common malignant tumor, it is necessary to conduct in-depth research on the pathogenesis, early diagnosis, and prognosis evaluation. The assessment of postoperative prognosis is beneficial to the accurate treatment for patients. Metabolomics can explore the metabolic changes in the process of cancer, to find new markers that can be used for early diagnosis of cancer and evaluation of efficacy.

Method: In this study, ultra-high-performance liquid chromatography quarter time of fly mass spectrometer (UPLC-Q-TOF-MS) technology was used to screen the metabolomics markers of blood samples from 24 CRC patients who underwent surgery and 24 healthy people. To verify the potential markers found above, At the same time, 8 CRC patients and normal control group were included.

Results: UDP-D-galactose, which shown significant concentration changed $2 \sim 3$ days after surgery was screened after statistical analysis. Moreover, the analysis of metabolic pathways in tissue samples also fully verified the correlation between UDP-D-galactose and CRC.

Conclusion: UPLC-Q-TOF-MS is a practical method for screening metabolic markers. Accurate monitoring the concentration of UDP-D-galactose in blood can provide important assistance for the treatment of CRC patients.

\section{Background}

Colorectal cancer is the third most common cancer worldwide, the fourth leading cause of cancer-related death in men, and the third leading cause of death among women [1]. Among cancer deaths in China, colorectal cancer ranks fifth, and its incidence is still rising [2].

The occurrence of colorectal cancer is the same as many other malignant tumors, it is affected by multiple genes and multiple factors. Therefore, the specific pathogenesis and mechanism of colorectal cancer are still under study. It had been reported [3] that abnormal miR-21 expression is associated with clinical manifestations in patients with CRC. Others [4] proposed that mutations of the tumor suppressor gene Adenomatous Polyposis Coli (APC) are critical in the development of colorectal cancer. Although numerous articles are published each year describing gene mutations or changes in gene expression levels associated with various cancers, gene-based tests are rarely used routinely in clinical settings [5]. Clinically, patients with colorectal cancer often lack typical clinical symptoms [6], and even some patients do not have any symptoms, so it often causes difficulty in diagnosis or even misdiagnosis. Among the current diagnostic methods, colonoscopy is the most authoritative test method. However, colonoscopy is highly invasive and has the disadvantage of being inconvenient and expensive [7]. Although clinical carcinoembryonic antigen (CEA) and fecal occult blood test (FOBT) are commonly used as tumor markers, the sensitivity and specificity are poor, which limit their applications [8]. Therefore, the search for blood molecular biomarkers, the use of blood testing to diagnose and postoperative follow-up treatment of CRC patients is of great significance. 
During the development of cancer, some tissue metabolites exhibit regular changes. Systematic application of analytical techniques to assess biological samples from healthy and diseased tissues, or metabolic levels in tissue samples obtained under other different conditions can provide us with indicators for detecting cancer progression [9].

Metabolomics is a new technology developed this year that can be used to quantify changes in small molecules at various pathological, physiological, and external stimuli in biological samples [10]. It can detect and identify different metabolites through a comprehensive analysis of low molecular weight endogenous metabolites in biological samples, revealing the pathways and roles of different metabolites in biological systems under different conditions [11]. The status of patients is monitor and the prognosis and treatment of postoperative colorectal cancer are comprehensively evaluated based on changes reflected from the genome, transcriptome, and proteome [12]. Metabolomics research usually involves multiple steps such as sample preparation, data acquisition, data processing, and statistical analysis. Correct data collection, processing, and analysis are prerequisites for obtaining statistically significant results [13]. With the in-depth study of metabolomics, a variety of detection and analysis methods have been applied to metabolomics, such as nuclear magnetic resonance (NMR), gas chromatography-mass spectrometer (GC-MS), liquid chromatography-mass spectrometer (LC-MS), etc. [14]. In recent years, highperformance liquid chromatography (HPLC) or ultra-high-performance liquid chromatography (UPLC) combined with mass spectrometer (MS) has been widely used due to its high efficiency and sensitivity [15].

In this study, we applied UPLC-Q-TOF-MS technology to analyze the plasma samples from CRC patients before and after surgery and healthy human as well as tissues samples from CRC patients and healthy human. The metabolic markers were screened by principal component analysis (PCA) and partial least squares discriminant analysis (PLS-DA). The detailed experimental process was shown in Fig. 1. Preliminary speculation was of great significance and value for the monitoring of colorectal cancer.

\section{Method}

\section{Materials}

Acetonitrile (ACN) was purchased from Concord Technology Co. Ltd (Tianjin, China). Distilled water was produced by Milli-Q ultra-pure water system (Millipore, Billeria, USA).

\section{Study population and sample collection}

Access to human samples complied with both Chinese laws and the guidelines of the Tianjin Union Medical Center Ethics Committee, and written informed consent was obtained from the patients. 24 blood samples were collected from CRC patients undergoing colorectal resection and that were maintained under fasting conditions in the Department of Colorectal Surgery, the Tianjin Union Medical Center. These patients came from different places in China and had different social environments, dietary practices, and personal habits. The diagnosis of CRC was confirmed in all cases by histopathologic examination 
and immunohistochemistry. The preoperative blood samples were drawn at 7:00 o'clock on the second day before hospitalization, and the postoperative blood samples were drawn in $3 \mathrm{~d}$ after surgery. During 3 $\mathrm{h}$ after surgery, the patients were not given any blood transfusion or other transfusion (electrolytes, glucose). Meanwhile, 24 blood samples were also collected from heath volunteers. Eight volunteers with anal benign diseases were selected for anal surgery, and specimens of benign anal disease lesions were selected, including hemorrhoids without infection and edema, and normal tissues near the anal fistula. Exclusion criteria were: (1) pregnant of lactating female; (2) metabolic diseases, such as diabetes mellitus (DM), gout, hyperlipidemia (HL); (3) hematopathy, including all types of leukemia and anemia; (4) any symptom of acute diseases during the last 2 weeks, such as: febrile, cough, headache, nausea, vomit, stomachache, diarrhea, hematuria; (5) momentous stress reaction during the last 2 weeks, such as psychic trauma and a large area of empyrosis; (6) use of specific drugs during the last 3 weeks, such as antibiotics (penicillins, quinolones, torlamician), hormone (deoxycortisone, dexamethasone), nonsteroid anti-inflammatory drugs (NSAIDs: acenterine, fragrans, saridon, contac), among others;(7)Excluding acute perianal diseases, such as perianal abscess, incarcerated hemorrhoids, etc. All the information about these CRC patients and were summarized in Table 1.

\section{Sample preparation}

The blood samples were kept in room temperature for $30 \mathrm{~min}$, subsequently centrifuged at $3,000 \mathrm{rpm}$ for 10 min at $4^{\circ} \mathrm{C}$, the serum samples were stored at $-80^{\circ} \mathrm{C}$ until use. Plasma and colorectum tissue samples were thawed on ice. $200 \mu \mathrm{L}$ of ACN were added to the $200 \mu \mathrm{L}$ plasma then vortex-mixed for 1 minute. After centrifugation (12000 r/min, $5 \mathrm{~min}$ ), the supernatant was taken for UPLC-Q-TOF-MS analysis. 30-50 $\mathrm{mg}$ of tissue sample was accurately weighted on ice. To extract all the ingredients, the tissue samples were fixed in $1 \mathrm{~mL}$ pure water and grinded in a mortar. $300 \mu \mathrm{L}$ of $\mathrm{ACN}$ were added into $200 \mu \mathrm{L}$ of the grinding fluid then vortex-mixed for 1 minute, centrifuged (12000 r/min, $5 \mathrm{~min}$ ) and finally took the supernatant for UPLC-Q-TOF-MS analysis.

\section{UPLC-Q-TOF-MS analysis}

A Waters Acquity ${ }^{\mathrm{TM}}$ UPLC system (Waters, Milford, MA, USA), including a quaternary pump, an autosampler and a temperature controller, that was used for analyzing the prepared plasma and tissue samples. The chromatographic separation was performed on an ACQUITY UPLC BEHC18 column $(100 \times 2.1 \mathrm{~mm}$, I.D., $1.8 \mu \mathrm{m})$ purchased from Waters at a flow rate of $0.3 \mathrm{~mL} / \mathrm{min}$ and $5 \mu \mathrm{L}$ injection volume. The run time was $13 \mathrm{~min}$ for each sample. The target column temperature and target sample temperature were maintained at $45^{\circ} \mathrm{C}$ and $5{ }^{\circ} \mathrm{C}$, respectively. Water was used as solvent $\mathrm{A}$ and $\mathrm{ACN}$ was used as solvent $B$ to conduct the gradient elution. $1 \%$ B for the first $0.5 \mathrm{~min}, 1 \%-50 \% \mathrm{~B}$ from 0.5 to 2 min, $50 \%-100 \%$ B from 2 to $9 \mathrm{~min}, 100 \%-1 \%$ B from 9 to 11 min and finally held for two minutes to get ready for the next injection. The column eluent was directed to the mass spectrometry without spilting.

Mass analysis was carried out on a XevoG2 Q-TOF (Waters MS Technologies, Manchester, UK) with ESI source both in positive and negative mode. The desolvation gas rate was set at $800 \mathrm{~L} / \mathrm{h}$ at a temperature 
of $450{ }^{\circ} \mathrm{C}$, the cone gas rate was set at $50 \mathrm{~L} / \mathrm{h}$ and the source temperature at $120^{\circ} \mathrm{C}$. The capillary voltage and the cone voltage were set at $3000 \mathrm{~V}$ and $40 \mathrm{~V}$ in the positive ionization mode, and $2000 \mathrm{~V}$ and $40 \mathrm{~V}$ in the negative ionization mode, respectively. All analyses used the lockspray to ensure accuracy and reproducibility; the leucine encephalin was used as the lock mass ( $\mathrm{m} / \mathrm{z} 556.6306$ for positive ion mode; $\mathrm{m} / \mathrm{z} 554.6147$ for negative ion mode). Data was collected in centroid mode from $\mathrm{m} / \mathrm{z}$ 50 to $\mathrm{m} / \mathrm{z} 1000$ with a lockspray frequency of $15 \mathrm{~s}$, and data averaging over ten scans.

\section{Statistical analysis}

PCA analysis was first used to detect the grouping trend and outliers. OPLS-DA analysis was performed to understand the difference between the two selected groups. Furthermore, variable importance in the projection (VIP) was calculated in OPLS-DA model to screen the potential biomarkers. Compounds whose VIP value was more than 1.5 in negative ion mode and 5.5 in positive ion mode that was considered as potential metabolic biomarkers in tissue samples, whose VIP value was more than 1.5 in negative ion mode and 8.0 in positive ion mode that was considered as potential metabolic biomarkers in plasma samples as well. Besides, the Wilcoxon test was conducted to determine the significance of each metabolite using IBM SPSS Statistics 20 then Graphpad Prism 6 was used to map the data. All univariate tests were two-sided and the significant differences between two groups to be compared were based on the paired test $\left({ }^{*} P<0.05,{ }^{* *} P<0.01,{ }^{* \star *} P<0.001,{ }^{* \star \star *} P<0.0001\right)$.

\section{Screening and analysis of biomarkers of CRC}

The potential metabolic biomarkers were identified by the database of human metabolome database (HMDB, http://www.hmdb.ca/) and mapped into their biochemical pathways through metabolic enrichment and pathway analysis was based on database search Kyoto Encyclopedia of Genes and Genomes (KEGG, http://www.genome.jp/kegg/) and MetaboAnalysis 4.0 software.

\section{Results}

The representative TIC chromatograms of both positive and negative ion scanning modes taken from control group as well as CRC group of different retention time were displayed in Fig. S1. According to the peak height data, totally 59,878 peaks of positive ions and 2,244 peaks of negative ions were detected by MassLynx using the same acquiring method mentioned above. Similarly, differences displayed in the chromatograms between the normal group and CRC group were significant. Not only can we find plasma markers of colorectal cancer from these ion peaks, but the levels of these markers were not the same before and after colorectal cancer surgery (Table S1) which have been reported before [16].

PCA variable loading plot was showed in Fig. S2b, f. Each point in the figure represented the ion peak detected by UPLC-Q-TOF-MS in the plasma samples and displayed its retention time. Ion peaks in the center were those with small differences in samples, while those dispersed in the periphery were the ion peaks with statistical significance. PCA scores plot (Fig. S2a, e) showed the separation trend of ESI- and ESI+ model between CRC group and control group which indicated a certain tendency of separation (ESI-: 
$\mathrm{R} 2 \mathrm{X}=0.38, \mathrm{ESI}+\mathrm{R} 2 \mathrm{X}=0.68$ ). After that, taking the first predicted principal component (abscissa) and the first orthogonal principal component (ordinate) mapped OPLS-DA (Fig. S2c, g). Results showed that the CRC group has a more distinct separation trend from the normal group (ESI-: R2Y=0.99, Q2=0.99; ESI+: $\mathrm{R} 2 \mathrm{Y}=0.99, \mathrm{Q} 2=0.99)$. The above results demonstrated the difference in metabolites between the CRC and normal groups. Finally, by means of VIP value distribution (Fig. S2d, h), ion peaks whose VIP value larger than 8.0 and 1.5 were selected in positive and negative ion modes $\bigotimes$ respectively.

The metabolic profiles of the tissue samples were performed by UPLC-Q-TOF-MS. The representative TIC chromatograms of both positive and negative ion scanning modes taken from control group as well as CRC group of different retention time were displayed in Fig. S3. According to the peak height data, totally 37,375 peaks of positive ions and 1,326 peaks of negative ions were detected by MassLynx using the same acquiring method mentioned above. By comparing the chromatograms, we found that the peak shapes of control group and CRC group were very different, which indicated metabolic differences in tissue samples, and these substances were likely to become potential metabolic markers of tumors.

Here, PCA variable loading plot was showed in Fig. S4b, f. Each point in the figure represented the ion peak detected by UPLC-Q-TOF-MS in the tissue samples and displayed its retention time. Ion peaks in the center were those with small differences in samples, while those dispersed in the periphery were the ion peaks with statistical significance. PCA scores plot (Fig. S4a, e) showed the separation trend of ESI ${ }^{-}$and $\mathrm{ESI}^{+}$model between $\mathrm{CRC}$ group and control group which indicated a certain tendency of separation (ESI: $\left.\mathrm{R} 2 \mathrm{X}=0.46, \mathrm{ESI}^{+}: \mathrm{R} 2 \mathrm{X}=0.54\right)$. After that, taking the first predicted principal component (abscissa) and the first orthogonal principal component (ordinate) mapped OPLS-DA (Fig. S4c, g). Results showed that the CRC group has a more distinct separation trend from the normal group (ESI: R2Y=0.99, Q2=0.94; $E S I^{+}$: $\mathrm{R} 2 \mathrm{Y}=0.97, \mathrm{Q} 2=0.92$ ). The above results demonstrated the differences in metabolites between the CRC and normal groups. Finally, by means of VIP value distribution (Fig. S4d, h), ion peaks whose VIP value larger than 5.5 and 1.5 were selected in positive and negative ion modes $₫$ respectively.

\section{Discussion}

Through the VIP values in the OPLS-DA model combined with the HMDB database, the final screening for potential metabolic markers was shown in Table S2. 6 and 11 markers were screened in positive and negative ion mode, respectively. The mean values of plasma biomarkers in all samples were summarized by group of control, preoperative and postoperative. The concentrations of selected metabolites in plasma samples were compared to detect whether there were statistical differences between the selected blood markers before and after surgery. The heatmaps of these 17 substances in the control group, the preoperative group, and the postoperative group are shown in Fig. 2 (Fig. 2a for the positive ion mode and Fig. $2 b$ for the negative ion mode). Although their concentrations changed before and after the surgery, the statistical results show that only one of those 17 substances were statistically significant. This may be caused by the blood samples which were collected 2-3 days after the surgery, when the concentration of metabolites was relatively small. Among them, the statistically significant substance was UDP-Dgalactose. By comparing the concentration before and after surgery with that of the control group, we 
found that the concentration of the metabolic markers changed in a normal direction after surgery. The results showed that the potential metabolic markers UDP-D-galactose screened by PCA analysis from the control group and CRC group also had statistical differences before and after surgery, which is expected to be a sensitive indicator for judging the operation and prognosis.

Through the VIP values in the OPLS-DA model combined with the HMDB database, the final screening for potential metabolic markers was shown in Table S3, and 19 markers were screened in each positive and negative ion mode. These substances are highly correlated with important biochemical metabolic pathways such as energy metabolism, amino acid metabolism, lipid metabolism and nucleotide metabolism.

The more detailed heat map of the tissue sample between the control group and CRC group is shown in Fig. 3. It is more visualized to see the difference of metabolic markers screened out in tissue samples between two groups. In addition, the results of metabolic pathways are shown in Fig. $4 \mathrm{~b}$. We showed the metabolic pathways whose P value were less than 0.05 in Fig. 4a, they are purine metabolism, propanoate metabolism, butanoate metabolism, synthesis and degradation of ketone bodies, citrate cycle (TCA cycle), pyruvate metabolism, valine, leucine and isoleucine degradation, terpenoid backbone biosynthesis, lysine degradation, glycolysis/gluconeogenesis and glyoxylate and dicarboxylate metabolism. Combined with the advanced reports, the relationship between the potential colorectal cancer metabolic markers and cancer were discussed. Finally, we depicted the metabolic pathways of colorectal cancer tissues, as shown in Fig. 5.

In the positive ion mode, there were 18 different metabolites were screened totally while seven of them were cancer-related metabolites that have been reported: Succinyl-5-aminoimidazole-4-carboxamide-1ribose 5'-phosphate (SAICAR), riboflavin cyclic-4', 5'-phosphate (cFMN), Dihydroxyacetone phosphate (DHAP), lactosamine, phosphatidylserine, thiamine pyrophosphate, and creatine riboside. Among them, cyclic-4', 5'-phosphate and lactosamine have been reported to be closely related to colorectal cancer.

cFMN has been identified as an endogenous liver compound, probably decomposed by flavin adenine dinucleotide (FAD, also known as active vitamin B2) in alkaline medium. In patients with the methylenetetrahydrofolate reductase (MTHFR) TT genotype, there was a negative correlation between vitamin B2 (also known as riboflavin) intake and colorectal cancer, and the risk of adenoma appeared to be more pronounced [17]. Meanwhile, studies by Robin Myte show a negative correlation between plasma vitamin B2 concentration and CRC risk. This study also demonstrates for the first time that the transcription/translation of RFVT has undergone profound changes in CRC [18]. For lactosamine, studies have shown that the structure of polylactosamine is often characterized by different cell types and different stages, and polylactosamine may be a potential target for 5-fluorouracil (5-FU) responses in colon cancer cells. Therefore, it may be a novel drug target candidate for the treatment of colon cancer [19]. However, there are no reports on its metabolic regulation until now.

The remaining five substances also have reports on their relationship with tumors. SAICAR is an intermediate of the denovo synthesis pathway of purine nucleotides and a metabolite with higher content 
in proliferating cells. It induces pyruvate kinase $\mathrm{M}_{2}\left(\mathrm{PKM}_{2}\right)$ in vitro and in cells, playing an important role in abnormal cell metabolism and sustained proliferation as well [20]. It has also been reported that SAICAR can activate $\mathrm{PKM}_{2}$ in a dimeric form [21]. At the same time, the interaction of SAICAR-PKM 2 also promoted the survival of cancer cells under glucose limiting condition. DHAP is a biochemical molecule involved in many reaction pathways such as glycolysis and an intermediate product of glycolysis and glycolformulose. This metabolic pathway is highly correlated with tumorigenesis [22]. Phosphatidylserine (PS) is an essential component of all human cells and is present in the inner leaflets of the cell membrane [23]. PS mainly inhibits the biological process, function and malignant transformation of host cells by activating PS receptors on immune cells and inhibiting anti-tumor immune responses [24]. Thiamine pyrophosphate (TPP) is a coenzyme form of vitamin B1. During hypoxic stress, tumor cells adaptively up-regulate thiamine transport. Once ingested, thiamine pyrophosphate kinase-1 (TPK1) promotes rapid phosphorylation of thiamine to TPP [25]. Creatine riboside and $\mathrm{N}$-acetylneuraminic acid (NANA) levels in urine were used as biomarkers of non-small cell lung cancer (NSCLC).

In the negative ion mode, the screened biomarkers were more closely related to colorectal cancer. Seven of them are: Cyclic AMP, caffeoyl aspartic acid, triglyceride (TG), UDP-D-galactose (2-), adenosine monophosphate, diacylglycerol (DG) and Succinic acid. Succinic acid is an important intermediate metabolite in the tricarboxylic acid cycle (TCA) [26], Studies showed significant changes in urinary metabolism in patients with colorectal cancer, as well as differences between patients with early and late colorectal cancer. Aspartic acid and succinic acid decreased in colorectal cancer compared with control group [27]. Adenosine monophosphate (AMP), also known as adenine ribonucleotide, is a unit of RNA and a constituent of NAD and NADP [28]. AMP is an important part in DNA synthesis in cells. The increase of AMP in CRC tissues reflects the rapid division of CRC cells. AMP was significantly different between adenomatous polyps and CRC tumor tissues and was lower in CRC tissues [29]. CA19-9 in serum is a well-known tumor marker and is often used in clinical diagnosis of cancer, especially colorectal cancer. UDP-galactose is necessary for the synthesis of CA 19-9 [30]. For CRC patients, TG levels were significantly higher and significantly different in patients with different TNM stages [31]. In human colon cancer, adenylate cyclase (AC) activity and intracellular CAMP concentration were much lower than dermal fibroblasts. The differences were so large that AC activity and intracellular concentrations of CAMP might be biochemical markers that could be used to distinguish colon cancer from benign cells in tissue culture [32]. The above analysis indicates that all the above substances are closely related to colorectal cancer. More depth and specific research on these metabolites will help to better study of the pathogenesis of colorectal cancer and find more effective treatment methods.

\section{Conclusion}

In this study, we applied UPLC-Q-TOF-MS technology to conduct metabolomics analysis on plasma samples of CRC patients before and after operation, as well as tissue samples of CRC patients and healthy people. We have mapped the metabolic pathway by analyzing related metabolites in tissue samples, which is very important for us to understand and study the metabolic pathogenesis of CRC 
deeply. In the study of plasma samples, the concentration of the metabolic marker UDP-D-galactose changed significantly within a short time after surgery, so it is expected to be a very sensitive plasma metabolic marker to evaluate the surgical prognosis.

\section{Abbreviations}

CRC: Colorectal cancer, UPLC-Q-TOF-MS: Ultra-high-performance liquid chromatography quarter time of fly mass spectrometer, APC: Adenomatous polyposis coli, CEA: Carcinoembryonic antigen, FOBT: Fecal occult blood test, NMR: Nuclear magnetic resonance, GC-MS: Gas chromatography-mass spectrometer, LC-MS: Liquid chromatography-mass spectrometer, HPLC: High-performance liquid chromatography, UPLC: Ultra-high-performance liquid chromatography, MS: Mass spectrometer, PCA: Principal component analysis, PLS-DA: Partial least squares discriminant analysis, CAN: Acetonitrile, DM: Diabetes mellitus, HL: Hyperlipidemia, NSAIDs: Nonsteroid anti-inflammatory drugs, VIP: Variable importance in the projection, HMDB: Human metabolome database, KEGG: Kyoto encyclopedia of genes and genomes, SAICAR: Succinyl-5-aminoimidazole-4-carboxamide-1-ribose 5'-phosphate, DHAP: Dihydroxyacetone phosphate, FAD: Flavin adenine dinucleotide, MTHFR: Methylenetetrahydrofolate reductase, 5-FU: 5Fluorouracil, $\mathrm{PKM}_{2}$ : pyruvate kinase $\mathrm{M}_{2}$, PS: Phosphatidylserine, TPP: Thiamine pyrophosphate, TPK1: Thiamine pyrophosphate kinase-1, NANA: N-acetylneuraminic acid, NSCLC: non-small cell lung cancer, TG: Triglyceride, DG: Diacylglycerol, TCA: Tricarboxylic acid cycle, AMP: Adenosine monophosphate, AC: Adenylate cyclase.

\section{Declarations}

\section{Acknowledgements}

Not applicable.

\section{Funding}

This work was financially supported by Tianjin Natural Science Foundation (Youth project, No. 17JCQNJC13000), Tianjin Union Medical Center Foundation (No. 2016YJ029), the Fundamental Research Funds for the Central Universities $₫$ Nankai University(63191165) and the Research Funding of Tianjin Health Commission (No. 16KG154).

\section{Availability of data and materials}

All data are presented in the figures, tables and Electronic Supplementary Material files.

\section{Conflict of Interest}

The authors declare that they have no conflict of interest.

\section{Authors' contributions}


$X Z$ and LD designed the study. ZZ, WZ and LZ researched relevant references for th study. CX, YL, YY, CC, and $\mathrm{ML}$ collected the clinical samples. ZZ and LS performed the experimental work and wrote the manuscript. Both authors have contributed to the data analysis, discussion and interpretations of the results. Both authors have read and approved the final manuscript.

\section{Consent for publication}

Not applicable.

\section{Ethics approval and consent to participate}

All procedures performed in this study that involved human participants were approved by the Research Ethics Committee of Tianjin Union Medical Center (2016YJ029) and conformed to the ethical standards of the 1964 Helsinki Declaration.

All subjects were over 18 years of age and voluntarily signed informed consent forms.

\section{References}

1. Anania G, Resta G, Marino S, et al. Treatment of colorectal cancer: A multidisciplinary approach. J Gastrointest Cancer. 2019; 50(3): 458-468.

2. Ma YL, Liu WJ, Peng JY, et al. A pilot study of gas chromatograph/mass spectrometry-based serum metabolic profiling of colorectal cancer after operation Mol Biol Rep. 2020; 37(3): 1403-1411.

3. Hao JP, Ma A. The ratio of miR-21/miR-24 as a promising diagnostic and poor prognosis biomarker in colorectal cancer. Eur Rev Med Pharmacol Sci. 2018; 22(24): 8649-8656.

4. Aghabozorgi AS, Bahreyni A, Soleimani A, et al. Role of adenomatous polyposis coli (APC) gene mutations in the pathogenesis of colorectal cancer; current status and perspectives. Biochime. 2019; 157: 64-71.

5. Kamiyama $\mathrm{H}$, Noda $\mathrm{H}$, Konishi $\mathrm{F}$, et al. Molecular biomarkers for the detection of metastatic colorectal cancer cells. World. J. Gastroenterol. 2014; 20(27): 8928-8938.

6. Adelstein BA, Macaskill P, Chan SF, et al. Most bowel cancer symptoms do not indicate colorectal cancer and polyps: a systematic review. BMC Gastroenterol. 2011; 11: 65-74.

7. Farshidfar F, Weljie AM, Kopciuk KA, et al. A validated metabolomic signature for colorectal cancer: exploration of the clinical value of metabolomics. Br J Cancer. 2016; 115(7): 848-857.

8. Tan BB, Qiu YP, Zou X, et al. Metabonomics identifies serum metabolite markers of colorectal cancer. J Proteome Res. 2013; 12(6): 3000-3009.

9. Kaushik AK, DeBerardinis RJ. Applications of metabolomics to study cancer metabolism. Biochim Biophys Acta Rev Cancer. 2018; 1870(1): 2-14.

10. Yu LZ, Aa JY, Xu J, et al. Metabolomic phenotype of gastric cancer and precancerous stages based on gas chromatography time-of-flight mass spectrometry. J Gastroenterol Hepatol. 2011; 26(8): 
1290-1297.

11. Li SW, Xie LS, Du ML, et al. Association study of genetic variants in estrogen metabolic pathway genes and colorectal cancer risk and survival. Arch Toxicol. 2018; 92(6): 1991-1999.

12. Wang XJ, Lv HT, Zhang AH, et al. Metabolite profiling and pathway analysis of acute hepatitis rats by UPLC-ESI MS combined with pattern recognition methods. Liver Int. 2014; 34(5): 759-770.

13. Rodrigues AM, Antonio C. Standard key steps in mass spectrometry-based plant metabolomics experiments: Instrument performance and analytical method validation. Methods Mol Biol. 2018; 1778: 19-31.

14. Zhang LJ, Chen B, Zhang JJ, et al. Serum polyunsaturated fatty acid metabolites as useful tool for screening potential biomarker of colorectal cancer. Prostaglandins Leukot Essent. Fatty Acids. 2017; 120: 25-31.

15. Xiao JF, Zhou B, Ressom WH. Metabolite identification and quantitation in LC-MS/MS-based metabolomics. Trends Analyt Chem. 2012; 32: 1-14.

16. Ma YL, Liu WJ, Peng JY, et al. A pilot study of gas chromatograph/mass spectrometry-based serum metabolic profiling of colorectal cancer after operation. Mol Biol Rep. 2010; 37(3): 1403-1411.

17. Powers HJ. Interaction among folate, riboflavin, genotype, and cancer, with reference to colorectal and cervical cancer. J Nutr. 2005; 135(Suppl 12): 2960S-2966S.

18. Tutino V, Defrancesco ML, Tolomeo M, et al. The expression of riboflavin transporters in human colorectal cancer. Anticancer Res. 2018; 38(5): 2659-2667.

19. Gao LP, Shen L, Yu MY, et al. Colon cancer cells treated with 5-fluorouracil exhibit changes in polylactosamine-type N-glycans. Mol Med Rep. 2014; 9(5): 1697-1702.

20. Keller KE, Doctor ZM, Dwyer ZW, et al. SAICAR induces protein kinase activity of $\mathrm{PKM}_{2}$ that is necessary for sustained proliferative signaling of cancer cells. Mol Cell. 2014; 53(5): 700-709.

21. Yan M, Chakravarthy S, Tokuda JM, et al. Succinyl-5-aminoimidazole-4-carboxamide-1-ribose 5'phosphate (SAICAR) activates pyruvate kinase isoform $\mathrm{M}_{2}\left(\mathrm{PKM}_{2}\right)$ in its dimeric form, Biochemistry. 2016; 55(33): 4731-4736.

22. Keller KE, Tan IS, Lee YS. SAICAR stimulates pyruvate kinase isoform $M_{2}$ and promotes cancer cell survival in glucose-limited conditions. Science. 2012; 338(6110): 1069-1072.

23. Sharma B, Kanwar SS. Phosphatidylserine: A cancer cell targeting biomarker. Semin Cancer Biol. 2018; 52(Pt 1): 17-25.

24. Park M, Kang KW. Phosphatidylserine receptor-targeting therapies for the treatment of cancer. Arch Pharm Res. 2019; 42(7): 617-628.

25. Jonus HC, Hanberry BS, Khatu S, et al. The adaptive regulation of thiamine pyrophosphokinase-1 facilitates malignant growth during supplemental thiamine conditions. Oncotarget. 2018; 9(83): 35422-35438.

26. Sun M, Yang ZB. Metabolomic studies of live single cancer stem cells using mass spectrometry. Anal. Chem. 2019; 91(3): 2384-2391. 
27. Chen JL, Fan J, Yan LS, et al. Urine metabolite profiling of human colorectal cancer by capillary electrophoresis mass spectrometry based on MRB. Gastroenterol Res Pract. 2012; 2012: 125890. doi: 10.1155/2012/125890.

28. Jung $\mathrm{K}$, Hong SH, Ngo HPT, et al. Crystal structures of an atypical aldehyde dehydrogenase having bidirectional oxidizing and reducing activities. Int. J. Biol. Macromol. 2017; 105(Pt 1): 816-824.

29. Huang YT, Lin YW, Chiu HM, et al. Curcumin induces apoptosis of colorectal cancer stem cells by coupling with CD44 marker. J Agric Food Chem. 2016; 64(11): 2247-2253.

30. Isshiki S, Togayachi A, Kudo T, et al. Cloning, expression, and characterization of a novel UDPgalactose: $\beta$-N-Acetylglucosamine $\beta 1,3-G$ alactosyltransferase ( $\beta 3 \mathrm{Gal}-\mathrm{T} 5$ ) responsible for synthesis of type 1 chain in colorectal and pancreatic epithelia and tumor cells derived therefrom. J Biol Chem. 1999; 274(18), 12499-12507.

31. Zhang X, Zhao XW, Liu DB, et al. Lipid levels in serum and cancerous tissues of colorectal cancer patients. World J Gastroenterol. 2014; 20(26): 8646-8652.

32. Nelson RL, Holian O. Adenylate cyclase activity and cyclic adenosine monophosphate levels in colon cancer lines and dermal fibroblasts and the effects of cholera toxin and epidermal growth factor. J. Surg. Oncol. 1988; 38(2): 108-112.

\section{Table}

Table 1. Clinical information and characteristics of human subjects

\begin{tabular}{|c|c|c|c|c|c|}
\hline & \multicolumn{3}{|l|}{ Blood Samples } & \multicolumn{2}{|l|}{ Tissue samples } \\
\hline & Control & Preoperation & Postoperation & Control & CRC \\
\hline Sample size & 24 & 24 & 21 & 8 & 22 \\
\hline Male/female & $16 / 8$ & $15 / 9$ & $13 / 8$ & $2 / 6$ & $13 / 9$ \\
\hline $\begin{array}{l}\text { Age (mean, } \\
\text { range) }\end{array}$ & $52.13(14 \sim 81)$ & $\begin{array}{l}58.75 \llbracket 39- \\
76 \rrbracket\end{array}$ & $59.48(39-73)$ & $62.38(48 \sim 72)$ & $63.09(30 \sim 80)$ \\
\hline \multicolumn{6}{|l|}{ Stage } \\
\hline TNM-I & - & $1(1 / 0)$ & $1(1 / 0)$ & - & $1(1 / 0)$ \\
\hline TNM-II & - & \multicolumn{2}{|c|}{$13(8 / 5) 12(7 / 5)$} & - & $12(9 / 3)$ \\
\hline TNM-III & - & \multicolumn{2}{|l|}{$10(6 / 4) 8(5 / 3)$} & - & $9(3 / 6)$ \\
\hline
\end{tabular}

\section{Figures}




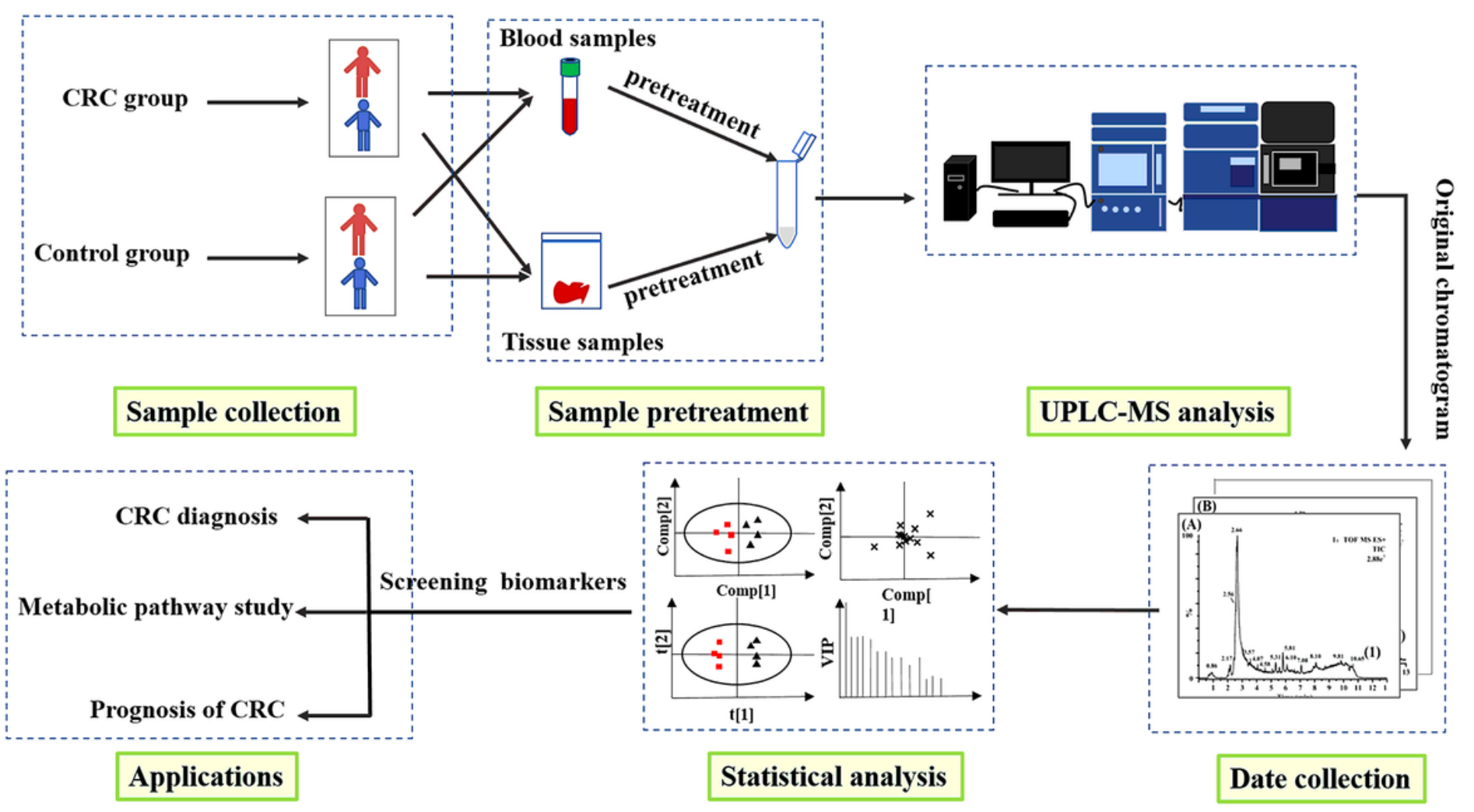

\section{Figure 1}

The overall flowchart of application of metabolomic method in this study. 
a
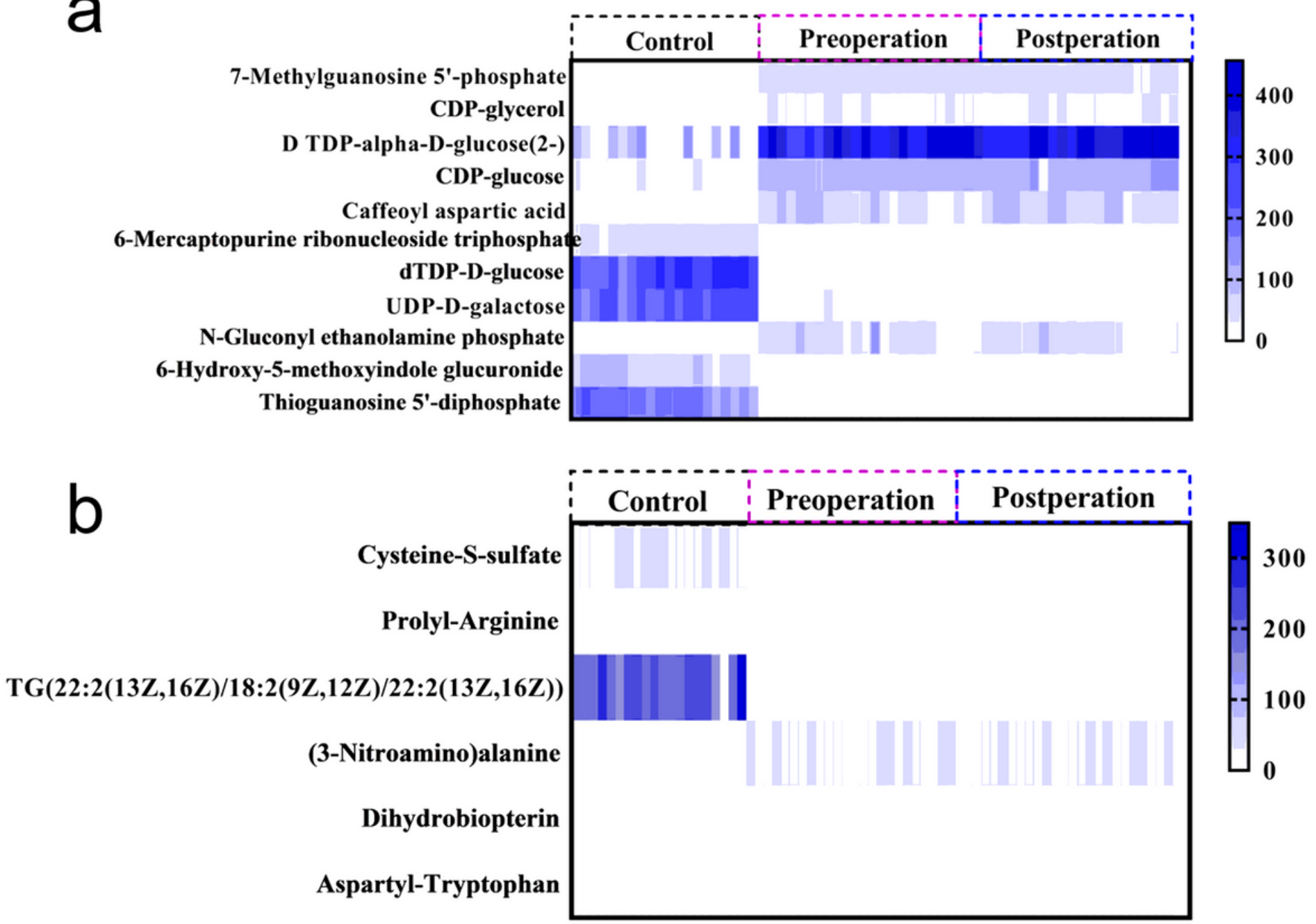

Figure 2

Heatmaps of the markers screened by $\mathrm{ESI}+(\mathrm{a})$ and $\mathrm{ESI}-(\mathrm{b})$ ion mode in the control group, the preoperative group, and the postoperative group. 
a

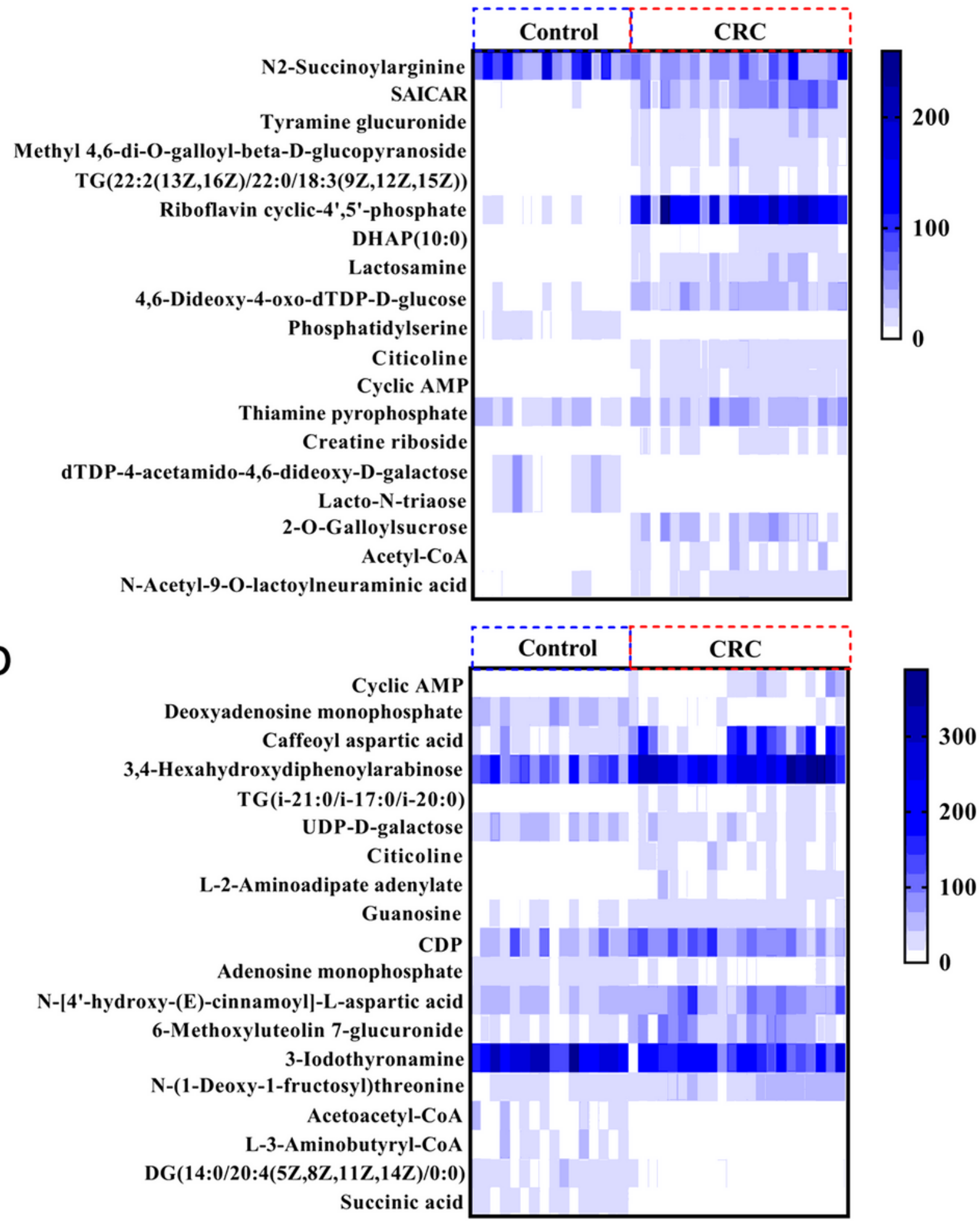

Figure 3

Heatmaps of the markers screened by ESI+(a) and ESI-(b) ion mode in the control group and the CRC group of tissue samples. 
a

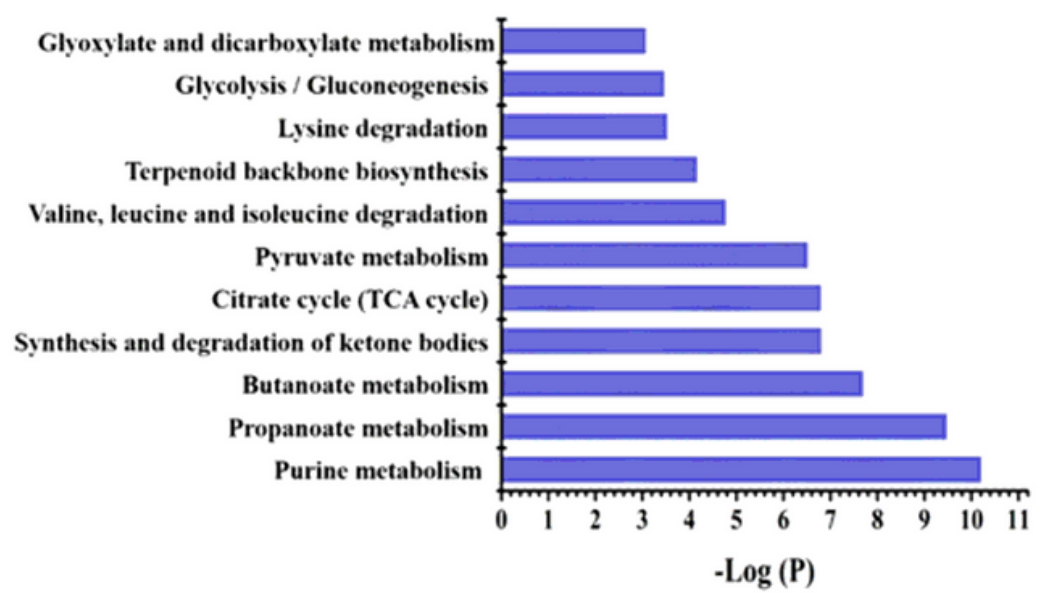

b

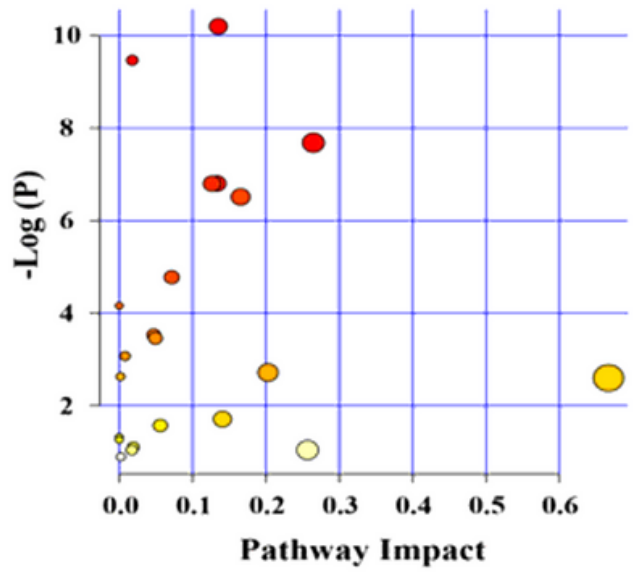

\section{Figure 4}

Log P value map(a) and metabolic pathway analysis chart(b) of candidate markers.

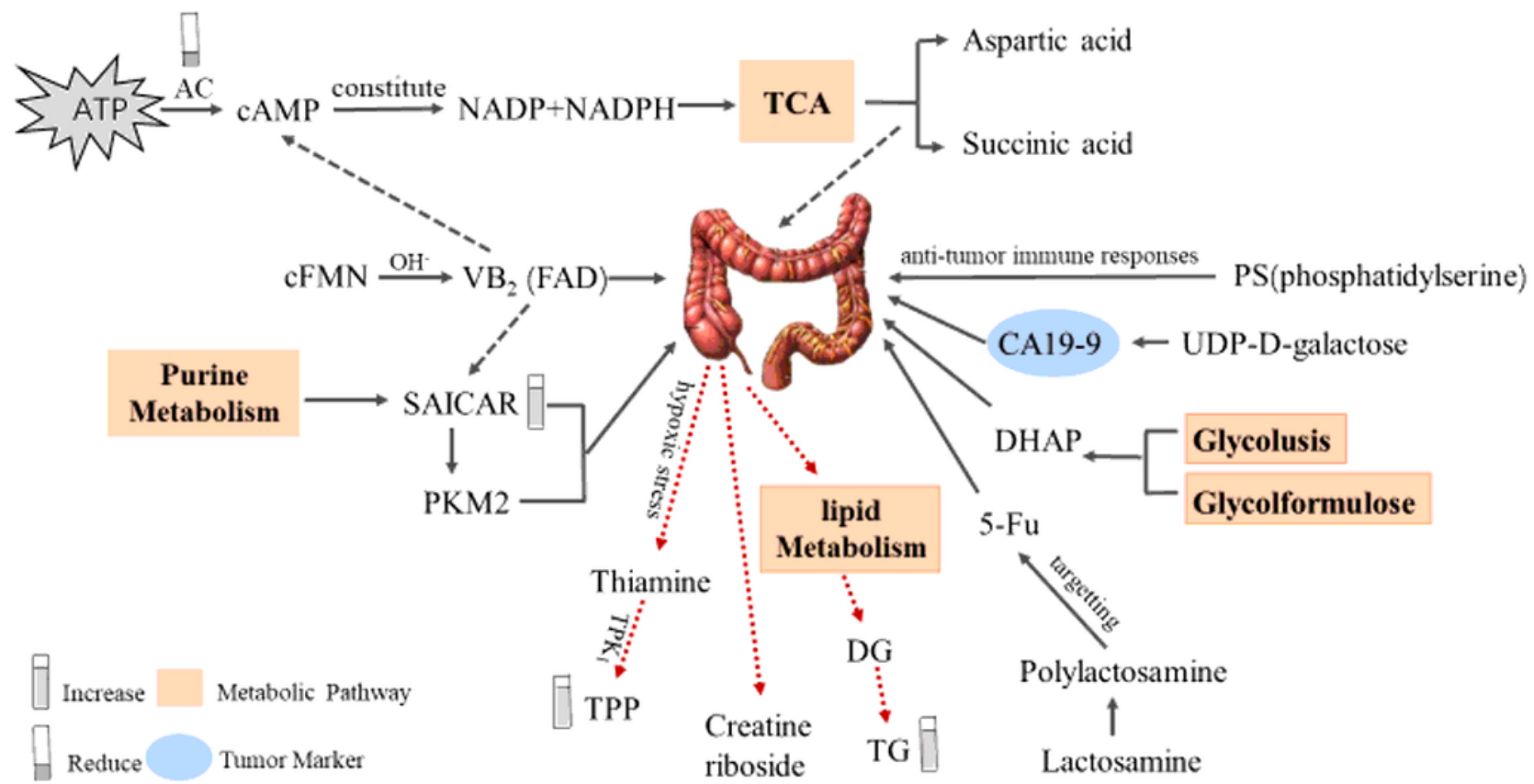

Figure. Metabolic pathways of colorectal cancer metabolic markers in tissue samples

\section{Figure 5}

Metabolic pathways of colorectal cancer metabolic markers in tissue samples.

\section{Supplementary Files}


This is a list of supplementary files associated with this preprint. Click to download.

- ElectronicSupplementaryMaterial.docx 